\title{
Les TIC au service du développement territorial dans la construction des savoirs
}

Marie-Michèle Venturini

\section{(2) OpenEdition}

12 Journals

Édition électronique

URL : http://journals.openedition.org/communicationorganisation/3254

DOI : 10.4000/communicationorganisation.3254

ISSN : $1775-3546$

Éditeur

Presses universitaires de Bordeaux

Édition imprimée

Date de publication : 1 juin 2005

Pagination : 164-173

ISSN : 1168-5549

\section{Référence électronique}

Marie-Michèle Venturini, « Les TIC au service du développement territorial dans la construction des savoirs », Communication et organisation [En ligne], 27 | 2005, mis en ligne le 07 juin 2012, consulté le 03 mai 2019. URL : http://journals.openedition.org/communicationorganisation/3254 ; DOI : 10.4000/ communicationorganisation.3254

Ce document a été généré automatiquement le 3 mai 2019.

(C) Presses universitaires de Bordeaux 


\title{
Les TIC au service du développement territorial dans la construction des savoirs
}

\author{
Marie-Michèle Venturini
}

1 Pendant des décennies, la qualité de la formation disponible et accessible sur un territoire était mise en avant comme facteur d'attractivité de ce territoire. Il s'agissait de la promesse implicite faite aux entrepreneurs de trouver localement de la main d'œuvre bien formée, disponible et aux acteurs locaux de trouver un emploi en relation avec les besoins de ces entreprises. Mais l'offre de formation présente sur un bassin ne dépend pas directement des pouvoirs locaux, dans le pire des cas ne rencontre pas toujours un intérêt démesuré de la part de ces mêmes acteurs d'une part et d'autre part, le développement des N.T.I.C autorise les mobilités et la virtualisation des échanges pédagogiques rompant ainsi, au moins en théorie, la relation avec le territoire.

2 Ces quelques phrases posent en préambule le cadre complexe dans lequel nous allons évoluer tout au long de cet article. Nous l'avons souligné, la complexité de notre objet, le territoire-éducatif est renforcée par la diffusion des N.T.I.C au sein des territoires. Nous pouvons désigner ce préambule par un mot: la territorialité ou l'appropriation des ressources d'un espace par les acteurs locaux dans une perspective de valorisation. L'appropriation des ressources éducatives mises à disposition traduit la création de contenus pédagogique territoriaux qui relèvent des processus de communication formalisés par un Distic ${ }^{1}$.

\section{Territoire éducatif et logique de proximité}

Pourtant, a priori le «local» n'a pas la réputation de favoriser la hauteur de vue qui convient pour comprendre les problèmes difficiles, l'horizon restreint des phénomènes tronquerait ou fausserait les analyses (Leroy, 2005). Ainsi, une approche locale des problèmes empêcherait une compréhension globale, offrant à l'observateur une réalité 
trop restreinte. Cette critique du «local», souvent associé à l'étroitesse de vue et à l'esprit partisan, ne semble pas s'adapter au système éducatif, lorsqu'on le définit par des opérations concrètes cherchant à répondre à l'ensemble des besoins de formation d'un public. Dans ce cas, le législateur affirme la nécessité d'une logique de proximité autant pour l'analyse des besoins que de la mise en œuvre des ressources éducatives qui y répondent. Cette logique de proximité se concrétisant aisément dans l'espace géographique commun de vie des acteurs où l'offre et la demande se rejoignent dans une recherche d'efficacité des actions de chacun. Ainsi, le local serait la bonne dimension pour une politique « d'aménagement du Territoire éducatif » parce qu'il favoriserait, à la fois deux conditions d'efficacité de la réponse aux besoins.

4 Tout d'abord la prise en compte de l'ensemble des ressources éducatives dans leur étendue, leur complémentarité, leur développement cohérent et concerté ... pour apprécier réellement la capacité d'action collective vers les objectifs d'éducation et de formation.

5 Ensuite la capacité à gérer l'hétérogénéité des besoins, ce qui sous-tend à la fois la capacité à favoriser leur expression et à construire des réponses adaptées. Ainsi, le Territoire restreint favorise l'analyse de la demande et offre une possibilité d'une réponse globale qui intègre aussi bien les dimensions éducatives du niveau scolaire de la formation technique, que des autres niveaux de l'insertion sociale et professionnelle (santé, culture, emploi etc. ...).

6 On comprend ainsi l'expression « penser globalement pour agir localement ». La réponse peut-être "globale » parce que le Territoire restreint permet d'envisager la mobilisation de tous les acteurs en utilisant les compétences spécifiques de leur travail en réseau.

7 A partir des définitions de l'échange et de l'action de développement territorial plusieurs questions nous viennent à l'esprit :

8 Quel est l'apport des T.I.C dans la construction des savoirs pour le développement territorial?

9 Comment doit-on organiser l'échange au niveau de la recherche scientifique afin de favoriser le développement territorial?

En somme, en quoi et comment ces outils favorisent-ils le développement territorial ?

11 Nous pouvons avancer quelques éléments de réponse pour faciliter et mettre en œuvre le maillage territorial ainsi que le partenariat public-privé afin de réunir les conditions d'un développement et de maintien des populations.

\section{Au sein des Universités}

12 Tout d'abord par l'implantation de filières de formations aux métiers dits numériques, aux outils d'informations scientifiques et techniques (URFIST par exemple) au sein des universités.

\section{Entre les centres universitaires}

13 Ensuite par la mise en place d'un projet de réseau d'universités de la communication. Ce qui aurait l'avantage de connecter ces sites universitaires à des réseaux existants. 


\section{Entre les acteurs du développement}

14 Par la création d'incubateurs d'entreprises au sein des universités pour favoriser le rapprochement des centres de recherche et des acteurs économiques d'une part et permettre à des étudiants de formuler, formaliser leur projet de création d'entreprise d'autre part.

\section{Au centre des collectivités}

Par la création de véritables places numériques au sein de communes ou de toute autre collectivité territoriale afin de créer des lieux de convergence et d'animation territoriale pour la population.

\section{En maillage du territoire}

Par la création de points d'accès à l'Internet citoyens à l'instar des points ERIC en région PACA. Ces points d'accès peuvent offrir outre l'accès à des ressources informatiques, la connexion à l'Internet, l'initiation de publics ciblés et l'offre de services publics.

17 Enfin, par l'implication toujours plus constante dans les différents projets territoriaux concernant les T.I.C (Cf. $\left.\mathrm{ADEC}^{2}\right)$ ou incluant les T.I.C comme outils essentiels à la bonne marche du projet (ex. : aide à la mise en place du tri sélectif dans certaines régions, utilisation de l'audiovisuel pour la promotion de certains domaines d'activités : film institutionnel du Centre d'Amélioration du Logement, film documentaire pour la défense de l'eau dans le monde ou encore sur les métiers de l'agriculture ...) . En fait, nous souhaitons établir cette relation circulaire du lien et de boucle entre une activité de recherche en développement territorial et une activité de porteur de projet pour un territoire donné. L'objectif ultime étant de doter les échelons territoriaux du capital formel préalable à leur valorisation puis à leur spécialisation en centre de compétences et de ressources.

\section{Fondements et supports de nos propositions :}

La démarche que nous préconisons et ses axes s'insèrent dans le programme de recherches M.A.I.N.A.T.E (Management de l'Information Appliquée au Territoire) initiée en 1996 au sein du laboratoire LePont de l'Université de Toulon et du Var. L'objet principal de ce programme est de pouvoir mesurer le réservoir de capacité de développement local que possède ou pas un territoire. Nos recherches visent les territoires de villes ou bassins de taille moyenne qui souhaitent définir et mettre en œuvre un projet de développement par l'intégration des T.I.C.

\section{L'intégration du système « territoire » dans un méta-modèle.}

Compte tenu de notre objet, le territoire, et de ses caractéristiques, il nous a semblé approprié de proposer un modèle du système «territoire » qui rende compte à la fois de la matérialité des objets territoriaux, des approches cognitives différentes des intervenants qui en effectuent une lecture spécifique, et du sens «territorial» qui 
transforme l'espace en ressources partagées (Oinas, Van Gils, 2001), (Steiner, Hartmann, 2001). Ainsi, nous avons eu recours dans nos travaux à un modèle qui se décompose en trois plans fortement imbriqués et indissociables les uns des autres. Nous l'avons nommé méta-modèle parce qu'il repose sur l'intégration de plans différents dont le cumul influe, à notre sens, la capacité d'un territoire à formuler une politique de développement. Les plans qui composent ce méta-modèle sont les suivants : celui de la matière physique (premier niveau), celui de l'information (deuxième niveau), celui de l'identité (troisième niveau). Nous pensons que ce modèle peut être aussi une représentation de la complexité d'un système territorial. Deux types d'approches complémentaires caractérisent ce métamodèle :

Une approche descriptive s'appuyant sur une description en trois niveaux irréductibles: celui des objets physiques, celui de l'information quantitative et qualitative, celui du tout ou de l'émergence.

Une approche dynamique rendant compte de l'évolution du système dans le temps. Le fonctionnement de cette entité repose sur l'interaction de nombreux acteurs directs et indirects qui restent à mobiliser.

Nous pensons qu'une politique de développement local relève de la médiation et suppose la capacité des acteurs à négocier dans la perspective de la définition d'un projet territorial. Pour prétendre à l'appropriation territoriale, les acteurs locaux ont besoin d'une structure pour s'entraîner à négocier puis s'engager envers les objectifs annoncés. Mais cette structure, réelle ou virtuelle, de reconstruction ou de valorisation des expériences locales ne peut être porteuse que si les membres en partagent les objectifs, possèdent les qualités requises pour mener à bien ce type de politique (Glize, Gleizes, Camps, 1998). Dès lors, l'espace à développer est à appréhender comme un ensemble de lieux, chacun doté de caractéristiques propres et un ensemble de flux qui relient les lieux et les constituent en système interdépendant (Savy, 1999). Cette définition parmi d'autres met l'accent sur les interactions entre les individus, les groupes informels et la structure organisationnelle, vecteur d'identité du territoire. Par identité, nous entendons la capacité de réponse à la banalisation des lieux, la dilution des territoires, la dissolution du local dans un mouvement de mondialisation. L'analyse des flux a des vertus singulières : elle dévoile les différenciations, les coopérations et les concurrences entre les lieux (Savy, op. cit.) et les acteurs. En premier objet, nous nous sommes préoccupés à représenter les liens des acteurs locaux et ce, par niveau indépendant sans chercher à établir des relations entre les niveaux différents du méta-modèle. C'est cette démarche que nous présentons dans la section suivante.

\section{La valorisation territoriale : une démarche transversale.}

Lorsqu'un échelon territorial réfléchit aux orientations futures de son avenir, il engage de fait un acte de développement. C'est-à-dire qu'il initie un processus de recherche de compétitivité globale (Coburn, 1999) . Il ne s'agit pas moins de renforcer la capacité d'attractivité du territoire, à le doter d'arguments spécifiques, à les faire connaître à des partenaires potentiels lorsqu'ils existent, éventuellement à manifester une volonté d'associer des partenaires à ce programme de développement. Ce processus relève, à notre sens, de la capacité d'adoption par des acteurs locaux très différents d'un objectif et d'une démarche (Boullier, 2000). Dans cette optique, le plan de développement devient acteur et outil pédagogique. Il est aussi un moyen d'interpénétrer les cultures 
différentes : entrepreneurs, institutionnels, éducatifs. Ce mouvement est donc fondé sur le décloisonnement, une approche transversale qui repose sur quelques piliers essentiels : et des compétences des autres, c'est-à-dire développer l'aspect grégaire de notre espèce et ce pour tendre vers le but qui est d'améliorer notre condition de vie et aussi pour répondre à une autre particularité de notre espèce : l'aspiration à plus de pouvoir. Cette définition peut selon nous tout à fait s'adapter à la notion de territoire puisque les collectivités gérant nos territoires ont découvert que la communication était un instrument de pouvoir et qu'elle était aussi une forme d'action ${ }^{3}$. En effet, la communication est devenu un instrument, un outil de gouvernement local c'est-à-dire que le rôle de la diffusion d'informations officielles s'est rapidement transformé en une véritable communication politique. Le territoire est l'objet d'un véritable jeu de pouvoir lié à l'appropriation des ressources de l'espace. Cette définition du territoire laisse entrevoir que le pouvoir n'appartient pas uniquement à ceux qui nous gouvernent mais aussi et surtout à l'ensemble des acteurs sociaux. On peut donc parler de politique territoriale concertée qui engage les acteurs dans une coordination territoriale. Cette coordination est un processus qui rassemble les acteurs et les transforme en un acteur collectif. (Bertacchini, 2004).

27 A partir de cette proposition double, on peut se demander en quoi et comment les T.I.C. favorisent-elles le développement territorial. Car plus qu'un système d'informations il s'agit de transformer l'information en connaissance et la connaissance en action (Polanco, 1999) pour que le territoire évolue. Notre objectif est de ce fait de favoriser la mise en réseau d'universités et de collectivités voire même d'entreprises, en somme de territoires et d'acteurs différents pour qu'ils deviennent des relais d'échanges scientifiques, technologiques, sociaux, politiques et économiques.

Les T.I.C. doivent être considérées comme des moyens, des outils appartenant à une politique de développement et relevant d'elle. En effet, l'accès et la maitrise des T.I.C. sont désormais l'un des facteurs de l'attractivité et de la compétitivité d'un territoire. Le développement d'un territoire doit forcément passer par l'appropriation des T.I.C. mises au service d'un patrimoine commun des connaissances. De nombreux chercheurs ont avancé la thèse de la gouvernance, c'est-à-dire un nouveau projet de l'action interactive pour gérer la société humaine. D’autres ont suggéré la thèse de "l'intelligence collective » qui permet grâce aux T.I.C. et aux réseaux, de promouvoir un nouvel 
humanisme garantissant à tous l'accès au savoir (Lévy, 1997). Enfin, d'autres soutiennent la théorie de l'empowerment qui développe l'idée d'une plus grande participation des citoyens dans la gestion et le contrôle des médias.

D'une façon générale et ce malgré les nuances et les argumentations spécifiques à chacune de ces thèses et d'autres, toutes ces idées convergent vers l'idée que les T.I.C. ne peuvent que favoriser la stratégie collective, la mutualisation des savoirs et surtout des actions.

Afin que le territoire se développe, il faut donc l'organiser selon plusieurs étapes et le doter du capital formel territorial évoqué par Bertacchini (2004) et résumer de la sorte :

- communiquer,

- s'informer, se former,

- échanger, transférer, afin de :

- valoriser,

- coopérer,

- d'innover.

Nous comprenons mieux ainsi comment les Tic peuvent, en respectant cette relation circulaire de double boucle, participer au développement territorial lors de la construction des savoirs.

\section{BIBLIOGRAPHIE}

Bakis H., Communication et territoires, Paris, La Documentation Française, 1990.

Beauchard J., (dir.), La Mosaïque territoriale. Enjeux identitaires de la décentralisation, La bibliothèque des territoires, L'Aube, 2003.

Bergeron P., Hiller C., « Competitive intelligence in Cronin, B. »,in : Annual Review for Information Science and Technology, vol. 36, 2001.

Bertacchini Y., « Entre information et anthropologie : le processus d'intelligence territoriale », in : Revue Humanisme et Entreprise, Les Cahiers du Centre d'Etudes et de Recherches, Paris, La Sorbonne Nouvelle, 2004.

Bonnet M., Desjeux D., (sous la dir. de), Les territoires de la mobilité, Paris, PUF, 2000.

Cardy Hélène, Construire l'identité régionale la communication en question, L'Harmattan, 1997. Champollion P., Piponnier A., « Première approche de la construction du processus qualité dans les sites Web interculturels », Actes du Colloque TICE et Méditerranée, in : International Journal of Information Sciences for Décision Making, $\mathrm{n}^{\circ}$ 18, 2004.

Coburn M., Competitive Technical Intelligence : A guide to Design, Analysis and action, Oxford University Press, 1999.

Flichy P., Quere L., (sous la dir.), «Communiquer à l'ère des réseaux», in : Réseaux n 100, Paris, Hermès, 2000. 
Glize P. Gleize M-P, Camps,V., « Une théorie de l'apprentissage fondée sur l'auto-organisation par coopération ", in : Apprentissage des méthodes naturelles aux méthodes articielles, Ed. Hermès, Paris, 1998.

Goux-Baudiment F., Quand les territoires pensent leur futur, L'Aube, 2001.

Jonas O., Territoires numériques, Ministère de l'Equipement, des transports et du logement, Paris, Certu, 2002.

Leroy Ph., «L'éducation au territoire et le concept de territoire éducatif : le défi pour le bassin d'éducation et de formation », Ouvrage collectif à paraître, Coll. Les ETIC, 2005.

Lévy P., L'intelligence collective, Pour une anthropologie du cyberspace, Paris, La Découverte, 1997.

Maherzi L., Rapport mondial sur la communication : les médias face aux défis des nouvelles technologies, Organisation des Nations Unies pour l'Education, Paris, UNESCO, 1997.

Miège B., La pensée communicationnelle, Coll. La communication en plus, Grenoble, Presses Universitaires de Grenoble, 1995.

Miège B., L'information-communication, objet de connaissance, De Boeck, 2004.

Moeglin P., (sous la dir. De), L'industrialisation de la formation. Etat de la question, Centre National de Documentation Pédagogique, 1998.

Pailliart I., Les territoires de la communication, Grenoble, Presses Universitaires de Grenoble, 1993.

Polanco X., «L'information scientifique et technique et l'outil Internet ? », Délégation aux systèmes d'information, Paris, CNRS, 1999.

Prax J-Y., Le management territorial à l'ère des réseaux, Editions d'Organisation, Paris, 2002.

Stevens J-F., Petit guide de prospective Nord-Pas-de-Calais 2020, L'Aube Nord, 2000.

Venturini M-M, «Entre verbe et projet : la médiation, fondation de l'échange et structure du développement local, un nejeu pour la Corse dans l'espace méditerranéen », in : International Journal of Information Sciences for Décision Making, $\mathrm{n}^{\circ}$ 16, 2004.

Vodoz L. et alii., NTIC et territoires, enjeux territoriaux des NTIC, Lausanne, CEAT, EPFL, 2002.

\section{NOTES}

1. Dispositif Socio-technique d'Information et de Communication.

2. Agence de Développement Economique de la Corse.

3. Venturini, 2004.

\section{RÉSUMÉS}

La formule utilisée en sous-titre et développée par les économistes, doit-elle s'appliquer à la formation lorsque celle-ci fait appel aux N.T.I.C et en quoi, c'est notre proposition, peut-elle 
contribuer à valoriser le territoire support de ces actions ? Pour y prétendre, elle doit synthétiser un discours complet et intégrer le fait que La vision statique du territoire a vécu. Le contexte urbain est bouleversé. Le brouillage des territoires et des références spatiales qui résulte de ces mobilités pose problème au politique et à tout acteur local dans la mesure où ceux-ci fonctionnent sur la délimitation territoriale de leurs compétences. Notre objet de compréhension et d'évaluation est le système territorial éminemment complexe dont chaque composante, chaque acteur, chaque intervenant est en totale interaction avec les autres et avec l'environnement, en même temps qu'il est ouvert sur un environnement extérieur spatial et temporel.

The traditionnal vision of the territory lived and the urban context is upset. Any kind of local actors, from citizens to business men through political, are facing to the jamming of territories and space references, which results from mobilities. Territorial development policies are based on limited geographic frontiers and so far, the local actors power frontiers are depending on. Invisible and virtual space takes an increasing weight. Public space is reduced less and less to a physical space supposing streets and places. Media space with the debates which it promotes cheek an increasingly important contemporary role and on the same time, territorial dynamics supposes a double communication: bottom up and signal down. Our object, the territorial educational system, is may be, the most eminently complex because, each component, each actor, each speaker is in total interaction with the others and the environment, at the same time as it is open on a space and temporal external environment. The main question and our purpose is how, to manage all these skills and transfer it.

\section{AUTEUR}

\section{MARIE-MICHĖLE VENTURINI}

Responsable pédagogique de la filière Communication-Europe et Méditerranée de l'Université de Corse. Chercheur associé au laboratoire I3M (Université de Toulon-Var). Mon domaine de recherche porte sur le développement territorial à partir de la construction des savoirs et de l'apport des TIC. Mail : mmventuri@univ-corse.fr 